\title{
Pulmonary metastasectomy is associated with prolonged survival among patients with bone and soft tissue sarcoma
}

\author{
JUNYA SHIMIZU $^{1}$, MAKOTO EMORI ${ }^{1}$, YASUTAKA MURAHASHI ${ }^{1}$, TOMOKO SONODA $^{2}$, \\ TAIJIRO MISHINA ${ }^{3}$, MASAHIRO MIYAJIMA ${ }^{3}$, ATSUSHI WATANABE ${ }^{3}$, SHINTARO SUGITA ${ }^{4}$, \\ KOHICHI TAKADA ${ }^{5}$, KAZUYUKI MURASE ${ }^{5}$, TADASHI HASEGAWA ${ }^{4}$ and TOSHIHIKO YAMASHITA ${ }^{1}$ \\ Departments of ${ }^{1}$ Orthopaedic Surgery, ${ }^{2}$ Public Health, ${ }^{3}$ Thoracic Surgery, ${ }^{4}$ Surgical Pathology and \\ ${ }^{5}$ Medical Oncology, Sapporo Medical University School of Medicine, Sapporo, Hokkaido 060-8543, Japan
}

Received July 17, 2019; Accepted February 5, 2020

DOI: $10.3892 / \mathrm{mco} .2020 .2009$

\begin{abstract}
The aim of the present study was to evaluate the survival impact of surgical resection among patients with pulmonary metastases from bone and soft tissue sarcomas. A total of 34 consecutive patients with $\leq 5$ pulmonary metastases from bone and soft tissue sarcomas were retrospectively reviewed. The patients included 19 men and 15 women, with a median age of 64.0 years and a median follow-up of 14.5 months. The oncological outcome was compared between patients who underwent surgical and non-surgical treatment. A total of 22 patients underwent surgery and 12 patients did not undergo surgery. The surgery group had 3 - and 5 year overall survival rates of 62 and 53\%, respectively. None of the patients in the non-surgery group survived to 3 years. Compared with the non-surgery group, surgery achieved significantly better 3 - and 5 year overall survival rates. Pulmonary metastasectomy was associated with significantly improved survival among patients who were aged $<64$ years $(\mathrm{P}=0.0155)$, as well as those who were aged $\geq 64$ years $(P=0.0444)$, which indicated that age was not associated with a difference in survival between the two groups. Therefore, pulmonary metastasectomy may improve the prognosis of patients with pulmonary metastases from bone and soft tissue sarcomas.
\end{abstract}

\section{Introduction}

Pulmonary metastasis (PM) is the leading cause of mortality among patients with bone and soft tissue sarcoma (BSTS). Furthermore, unresectable PM is associated with an extremely poor prognosis, with a median survival of 11 months (1). Surgical resection is a generally accepted treatment for PM,

Correspondence to: Dr Junya Shimizu, Department of Orthopaedic Surgery, Sapporo Medical University School of Medicine, West 16, South 1, Chuo-ku, Sapporo, Hokkaido 060-8543, Japan

E-mail: jshimizu@sapmed.ac.jp

Key words: lung metastasis, video-assisted thoracic surgery, bone and soft tissue sarcoma, pulmonary metastasectomy, clinical result with complete resection of PM achieving a survival of up to 33 months and 3 year survival rates of $30-42 \%(1,2)$. However, surgical management of PM has achieved important results in soft tissue sarcoma treatment, but without level I or II evidence (1). Approximately $25 \%$ of the patients are alive 5 years after surgery, but most studies do not reveal whether these patients survive with or without relapse (3). To the best of our knowledge, no randomized trials comparing survival after surgery and non-surgical treatment have been published to date (3). Furthermore, the populations from which patients were selected for pulmonary metastasectomy and the prognosis of unresected patients are seldom reported. Therefore, the aim of the present study was to retrospectively compare the prognosis of patients with PM from BSTS receiving surgical and non-surgical treatment.

Our preliminary study demonstrated that lung metastasectomy had the potential to improve patient prognosis (4). Thus, it was herein hypothesized that pulmonary metastasectomy may be associated with an improved prognosis in this setting, compared with non-surgical treatment. The present study compared patients with PM from BSTS according to whether they underwent surgical or non-surgical treatment, with the aim to identify factors that are predictive of a good outcome among the surgically treated patients.

\section{Patients and methods}

Patients. The present study retrospectively reviewed prospectively collected oncology/reconstruction surgery data and identified 56 consecutive patients ( 33 men and 23 women) with BSTS who developed PM between January 2005 and March 2017. The retrospective protocol of the present study was approved by the Institutional Review Board of Sapporo Medical University (Sapporo, Japan). Patients who participated in this research had complete clinical data. The signed informed consents were obtained from the patients or the guardians. All patients had complete local control of the primary tumor and underwent chest computed tomography (CT) as part of metastatic surveillance at 3-6 month intervals. PM was defined as a growing nodule that was detected during CT examination. Surgical staging was performed according to the seventh edition of the TNM system of the International 
Union Against Cancer (5). Histological grading was performed based on the system for soft tissue sarcoma from the French Federation of Cancer Centers (6), which is based on the tumor's mitotic index, extent of necrosis, and degree of histological differentiation. Data were collected for each patient regarding demographic characteristics (age and sex), primary tumor histopathology, metastasis characteristics (number and location), surgical approach, outcome, and interval between the primary treatment and PM diagnosis.

Treatment. The decision to perform pulmonary metastasectomy was based on the discretion of the attending thoracic surgeon after discussion. The eligibility criteria for pulmonary metastasectomy were as follows: i) The primary sarcoma had been completely resected, ii) there were $\leq 5 \mathrm{PM}$ nodules, iii) the pulmonary lesions were considered completely resectable, iv) the patient's general condition was sufficient to withstand surgery and v) the patient provided informed consent. The patients underwent video-assisted thoracic surgery (VATS) that was performed by several thoracic surgeons at our hospital, and the specific operative approach was selected based on the surgeon's judgement and the feasibility of R0 resection. All patients were carefully followed up using CT every 3-6 months after surgery. Whether to administer chemotherapy was decided through discussion with the oncologists.

Statistical analysis. Demographic characteristics and overall survival (OS) were compared between patients who underwent pulmonary metastasectomy or non-surgical treatment. The demographic and clinical characteristics were compared using Fisher's exact test or the Mann-Whitney U test, as appropriate. The OS outcomes were calculated from the metastasectomy or initiation of non-surgical treatment to the date of death or the date of the last follow-up. Kaplan-Meier curves were used to describe OS, and the results were compared using the log-rank test. All statistical data were analyzed using GraphPad Prism, version $5.0 \mathrm{f}$ (GraphPad Software Inc.) and $\mathrm{P}<0.05$ was considered to indicate statistically significant differences.

\section{Results}

Patients and characteristics. Between January 2005 and March 2017, 56 patients who developed PM from BSTS were identified. A total of 24 patients underwent pulmonary metastasectomy and 32 patients received non-surgical therapy. Patients with $>5$ PM nodules or missing data were excluded from the study (Fig. 1). The final analysis included 34 consecutive patients with a median age of 64 years (range, $14-89$ years) and a median follow-up of 14.5 months (range, 1-136 months). The most common histological type was osteosarcoma $(n=10)$, followed by leiomyosarcoma $(n=5)$, undifferentiated pleomorphic sarcoma $(n=5)$ and myxofibrosarcoma $(n=3)$. The patients' demographic and tumor characteristics are summarized in Table I. Of the 34 patients, 22 underwent surgery and 15 of those patients received chemotherapy. The remaining 12/34 patients did not undergo surgery. However, 10 of those patients received chemotherapy. Two patients did not receive any treatment (Table II). Patients who received non-surgical treatment were significantly older compared with patients who underwent pulmonary metastasectomy $(\mathrm{P}=0.0162)$.
Table I. Characteristics of all patients.

\begin{tabular}{lc}
\hline Characteristics & No \\
\hline Sex (male/female) & $19 / 15$ \\
Age, years (median/range) & $64.0 / 14-89$ \\
Location (extremity/trunk) & $25 / 9$ \\
Adjuvant chemotherapy & \\
Yes & 25 \\
No & 9 \\
Histology & \\
Osteosarcoma & 10 \\
Leiomyosarcoma & 5 \\
UPS/MFS & $5 / 3$ \\
Dedifferentiated liposarcoma & 3 \\
MPNST & 2 \\
Chondrosarcoma & 2 \\
Chordoma & 1 \\
Myxoid liposarcoma & 1 \\
Epithelioid sarcoma & 1 \\
Synovial sarcoma & 1 \\
Bone sarcoma/soft tissue sarcoma & $13 / 21$ \\
Grade of soft tissue sarcoma (I/II/III) & $4 / 3 / 14$ \\
\hline
\end{tabular}

UPS, undifferentiated pleomorphic sarcoma; MFS, myxofibrosarcoma; MPNST, malignant peripheral nerve sheath tumor.

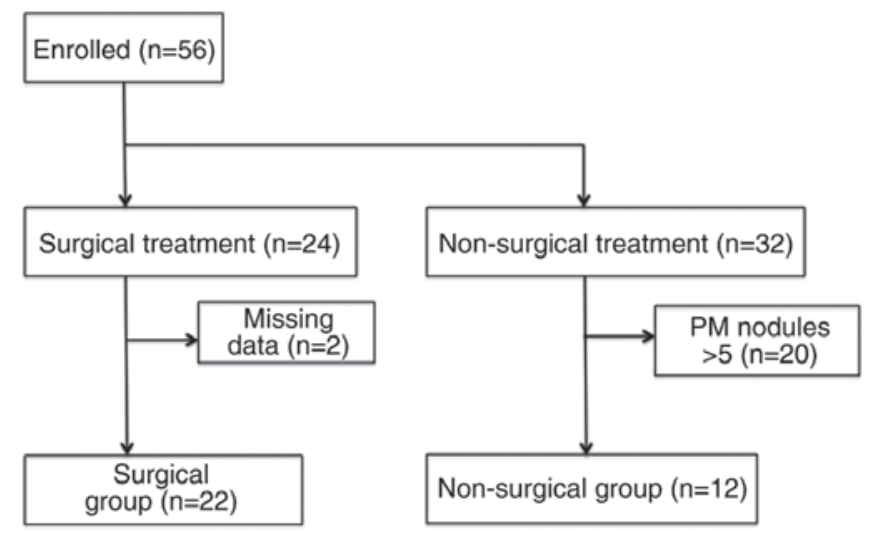

Figure 1. Flow chart of surgical treatment for lung metastasis. PM, pulmonary metastasis.

Survival. The OS curve for all patients (3 year OS, 48\%) is shown in Fig. 2. The univariate analyses failed to identify any demographic or clinical factors that significantly affected the prognosis among all 34 patients (Table I). Patients who underwent pulmonary metastasectomy had a median survival of 101 months, while the non-surgical group had a median survival of 11 months (Fig. 3). Compared with the non-surgical group, the pulmonary metastasectomy group had higher 3 and 5 year OS rates ( 0 vs. $62 \%$ and 0 vs. $53 \%$, respectively). The log-rank test confirmed that these differences were statistically significant $(\mathrm{P}=0.0029)$. Furthermore, pulmonary metastasectomy was associated with significantly improved 
Table II. Characteristics of patients according to whether they did (surgical group) or did not undergo pulmonary metastasectomy (non-surgical group).

\begin{tabular}{lccc}
\hline Patient characteristics & Non-surgical group & Surgical group & P-value \\
\hline No & 12 & 22 & NS \\
Sex (male/female) & $7 / 5$ & $12 / 10$ & 0.0162 \\
Age (years median/range) & $68.5 / 17-89$ & $49.5 / 14-81$ & NS \\
Location (extremity/trunk) & $7 / 5$ & $18 / 4$ & NS \\
Number of metastasis (median/range) & $2.0 / 1-5$ & $2.0 / 1-5$ & NS \\
Adjuvant chemotherapy & & & NS \\
Yes & 10 & 15 & \\
No & 2 & & \\
Histology & & 9 & \\
Osteosarcoma & 1 & 3 & \\
Leiomyosarcoma & 2 & 3 & \\
UPS/MFS & $3 / 2$ & 1 & \\
Dedifferentiated liposarcoma & 0 & 0 & \\
MPNST & 1 & 1 & NS \\
Chondrosarcoma & 2 & 1 & NS \\
Chordoma & 0 & 0 & \\
Mixoid liposarcoma & 0 & 1 & \\
Eplithelioid sarcoma & 1 & $10 / 12$ & \\
Synovial sarcoma & 0 & $2 / 1 / 9$ & \\
Bone sarcoma/soft tissue sarcoma & $3 / 9$ & & \\
Tumor grade of soft tissue sarcoma (Grade I/II/III) & $2 / 2 / 5$ & & \\
\hline
\end{tabular}

UPS, undifferentiated pleomorphic sarcoma; MFS, myxofibrosarcoma; MPNST, malignant peripheral nerve sheath tumor.

Table III. Characteristics of patients who underwent pulmonary metastasectomy.

\begin{tabular}{lc}
\hline Variables & $\begin{array}{c}\text { Overall } \\
\text { (Range) }\end{array}$ \\
\hline Patient number & 22 \\
Operative approach (VATS/open) & $22 / 0$ \\
Laterally (unilateral/bilateral) & $22 / 0$ \\
Type of resection (wedge/lobectomy/segmetal) & $17 / 3 / 2$ \\
Completeness of resection (R0/R1/R2) & $23 / 0 / 0$ \\
Hospitalization (days) & $8(5-15)$ \\
Complications & 1 \\
Pneumothorax & 1 \\
\hline
\end{tabular}

VATS, video-assisted thoracic surgery.

survival among patients who were aged $<64$ years $(\mathrm{P}=0.0155)$ as well as those who were aged $\geq 64$ years ( $\mathrm{P}=0.0444$; Fig. 4). This finding demonstrated that age did not affect the survival of patients who underwent surgical treatment.

Characteristics of the pulmonary metastasectomy group. A total of 22 patients underwent pulmonary metastasectomy via VATS, and their characteristics are summarized in Table III.

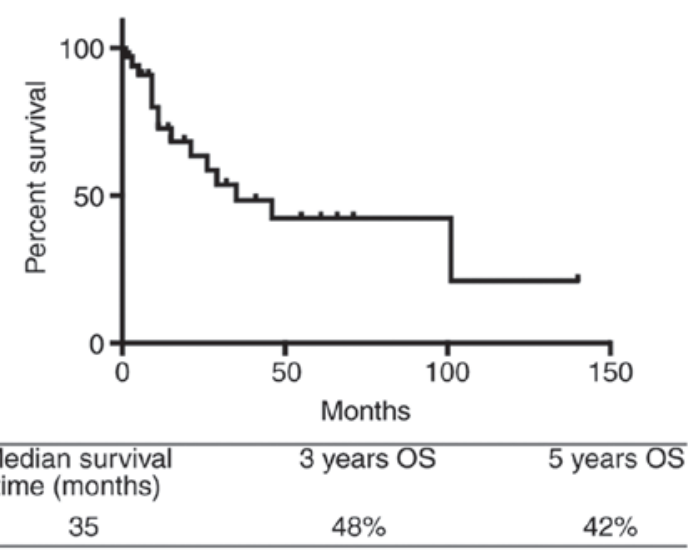

Figure 2. OS among all patients. OS, overall survival.

A total of 17 patients (77\%) underwent wedge resection, 3 patients (14\%) underwent lobectomy, and 2 patients (9\%) underwent segmental resection, with a median number of resected metastases of 2 (range, 1-5). R0 resection was achieved in all patients, and the median disease-free interval was 15 months. The mean hospital stay was 8 days (range, 5-15 days), and only 1 patient experienced pneumothorax as a postoperative complication. There were no postoperative fatalities. A total of 13 patients remained alive without disease after their first thoracic surgery, and 9 patients developed recurrence at a median interval of 9 months (range, 3-46 months). 
Table IV. Characteristics of patients who experienced poor and good outcomes.

\begin{tabular}{|c|c|c|c|}
\hline Variables & Poor outcome & Good outcome & P-value \\
\hline Patient no & 9 & 13 & \\
\hline Sex (male/female) & $4 / 5$ & $8 / 5$ & NS \\
\hline Age (median/range) & $48.0 / 15-82$ & $54.0 / 14-81$ & NS \\
\hline Number of metastasis (median/range) & $1.0 / 1-5$ & $2.0 / 1-5$ & NS \\
\hline Adjuvant chemotherapy & & & NS \\
\hline Yes & 6 & 9 & \\
\hline No & 3 & 4 & \\
\hline Location & & & NS \\
\hline Spine/Pelvic & 3 & 1 & \\
\hline Extremity & 6 & 12 & \\
\hline Pathology & & & NS \\
\hline Bone & 6 & 4 & \\
\hline Soft tissue & 3 & 9 & \\
\hline DFI (median/months) & $9 / 3-46$ & $19 / 2-66$ & NS \\
\hline
\end{tabular}

DFI, disease free interval.

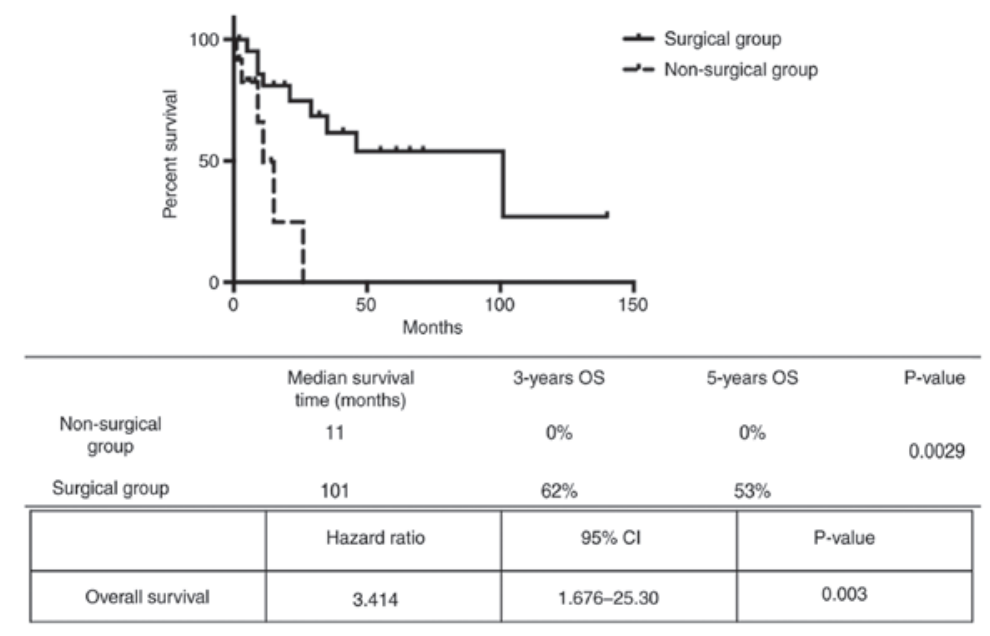

Figure 3. OS among patients who underwent pulmonary metastasectomy (surgical group) and those in the non-surgical group. The median survival of the surgical and non-surgical groups was 101 and 11 months, respectively, indicating that lung metastasectomy may prolong OS. CI, confidence interval; OS, overall survival.
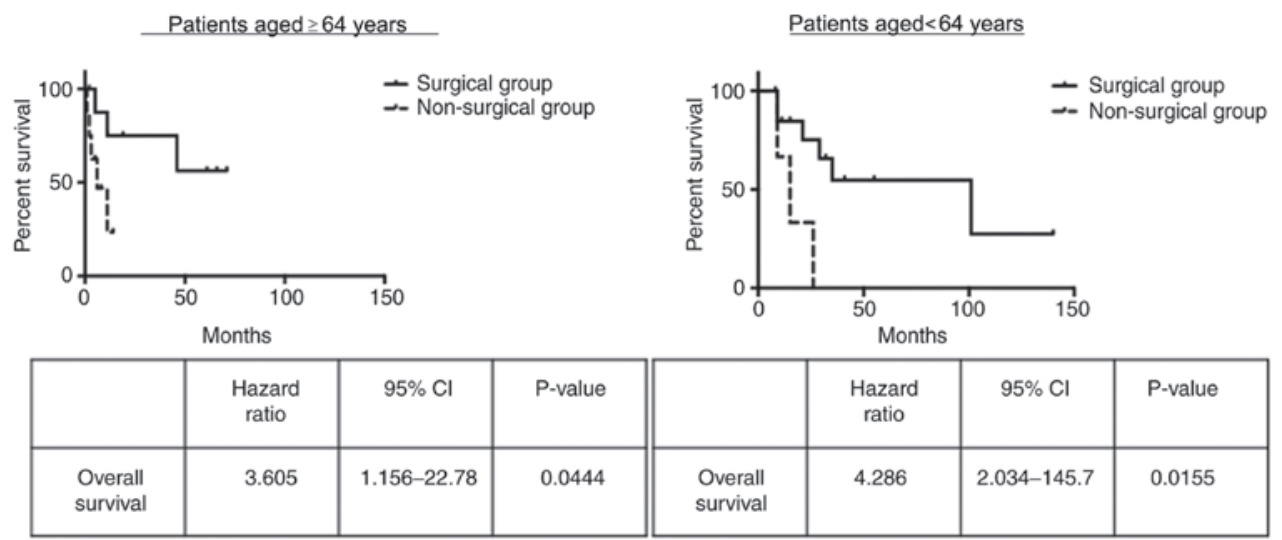

Figure 4. Pulmonary metastasectomy was associated with significantly better outcomes among patients who were aged years64 years as well as those who were aged $\geq 64$ years. Using the log-rank test, it was demonstrated that lung metastasectomy had significant beneficial effects on overall survival in patients aged $\geq 64$ and $<64$ years $(\mathrm{P}=0.0444$ and $\mathrm{P}=0.0155$, respectively). $\mathrm{CI}$, confidence interval. 
Analysis according to outcomes in the pulmonary metastasectomy group. The pulmonary metastasectomy group included 9 patients who succumbed to their disease during follow-up (poor outcome) and 13 patients who survived throughout the follow-up period (good outcome). Comparison of these subgroups failed to detect any significant predictors of poor or good outcomes (Table IV).

\section{Discussion}

Previous reports have suggested that pulmonary metastasectomy confers a survival benefit. Rusch reported that the 5 year OS rate was $17 \%$ among patients who underwent non-surgical treatment for PM from BSTS, whereas several other reports revealed 3 year OS rates of 28-35\% with resection of lung metastases and 5 year OS rates of $21-38 \%$ (7-10). Complete resections were performed in the majority of these cases, although certain reports included partial resections (7-11). However, this benefit has not been fully validated, as these reports did not analyze the oncological outcome between patients who underwent pulmonary metastasectomy or non-surgical treatment $(7,8)$. Therefore, we herein compared the prognoses between surgical and non-surgical patients with PM from BSTS to evaluate the survival impact of surgical resection. Patients with >5 PM nodules were excluded, as it has been reported that patients with $\leq 5$ nodules have a longer estimated median survival compared with those with $>5$ nodules (12). The present study revealed that pulmonary metastasectomy was associated with improved OS among patients with PM from BSTS. These outcomes are further supported by the fact that we attempted to identify PM as soon as possible via frequent follow-up pulmonary CT examinations, and that all our patients were treated using VATS, which allows for faster recovery and less pain.

The group of patients who underwent pulmonary metastasectomy was significantly younger compared with the non-surgical group, which may have been associated with their improved outcomes. However, we compared patients with a median age of $\geq 64$ and $<64$ years and found that the survival benefit of pulmonary metastasectomy persisted in both analyses. Thus, it appears that patient age did not significantly affect the association between pulmonary metastasectomy and outcome.

Several studies have examined prognostic factors among patients undergoing pulmonary metastasectomy for BSTS, which were reported to be complete resection, disease-free interval, PM size, PM number, and laterality (13-17). However, the present study failed to detect significant prognostic factors in the pulmonary metastasectomy group. This may be attributed to the fact that we evaluated prognosis based on whether the patients were alive with disease or had succumbed to their disease, without considering the follow-up period. It is also possible that the relatively small sample size affected the ability to detect significant prognostic factors.

There were certain limitations to the present study: i) The retrospective design and small sample size, which are associated with risks of bias, ii) selection bias was present, as patients who were unable to tolerate surgery did not undergo pulmonary metastasectomy, iii) the follow-up period was shorter compared with those in previous studies and iv) treatment was not performed in a randomized, controlled manner.
In conclusion, the findings of the present study revealed that, compared with non-surgical treatment, pulmonary metastasectomy was associated with improved prognosis in patients with PM from BSTS.

\section{Acknowledgements}

The authors would like to thank Dr Tamotsu Soma, Dr Mikito Sasaki, Dr Mitsunori Kaya and Dr Takuro Wada for orthopedic assistance, as well as Dr Tsukasa Hori, Dr Masaki Yamamoto and Dr Keita Igarashi for pediatric oncological assistance.

\section{Funding}

This study was partly supported by grants from JSPS KAKENHI (18K09112 to ME).

\section{Availability of data and materials}

The datasets generated and/or analyzed during the present study are available from the corresponding author on reasonable request.

\section{Authors' contributions}

JS wrote the manuscript, analyzed the data and performed the statistical analysis. ME interpreted patient data and wrote the manuscript. YM collected and interpreted patient data. TS performed statistical analysis. TM, MM and AW performed pulmonary metastasectomy. SS and TH made the pathological diagnoses. KT and KM were involved in administering the adjuvant chemotherapy. TY designed the present study and wrote the manuscript. All authors read and approved the final manuscript.

\section{Ethics approval and consent to participate}

The retrospective protocol of the present study was approved by the Institutional Review Board of Sapporo Medical University (Sapporo, Japan). Patients who participated in this research had complete clinical data. The signed informed consents were obtained from the patients or the guardians.

\section{Patient consent for publication}

Not applicable.

\section{Competing interests}

The authors declare that they have no competing interests.

\section{References}

1. Billingsley KG, Burt ME, Jara E, Ginsberg RJ, Woodruff JM, Leung DH and Brennan MF: Pulmonary metastases from soft tissue sarcoma: Analysis of patterns of diseases and postmetastasis survival. Ann Surg 229: 602-610, 1999.

2. Hamaji M, Chen F, Miyamoto E, Kondo T, Ohata K, Motoyama H, Hijiya K, Menju T, Aoyama A, Sato T, et al: Surgical and non-surgical management of repeat pulmonary metastasis from sarcoma following first pulmonary metastasectomy. Surg Today 46: 1296-1300, 2016 
3. Treasure T, Fiorentino F, Scarci M, Møller H and Utley M Pulmonary metastasectomy for sarcoma: A systematic review of reported outcomes in the context of thames cancer registry data. BMJ Open 8: e001736, 2012.

4. Shimizu J, Emori M, Yasutaka M and Yamashita T: Pulmonary metastasectomy is associated with a survival benefit among patients with bone and soft tissue sarcoma. J East Jpn Orthop Traumatol 31: 16-21, 2019 (In Japanese).

5. Sobin LH, Gospodarowicz MK and Wittekind C (eds): TNM classification of malignant tumors 7 th ed: International Union against Cancer (UICC). Vol 78. Elsevier BV, Netherlands, pp1278, 2010.

6. Coindre JM: Grading of soft tissue sarcomas: Review and update. Arch Pathol Lab Med 130: 1448-1453, 2006.

7. Schur S, Hoetzenecker K, Lamm W, Koestler WJ, Lang G, Amann G, Funovics P, Nemecek E, Noebauer I, Windhager R, et al: Pulmonary metastasectomy for soft tissue sarcoma-report from a dual institution experience at the medical university of vienna. Eur J Cancer 50: 2289-2297, 2014

8. Rehders A, Hosch SB, Scheunemann P, Stoecklein NH, Knoefel WT and Peiper M: Benefit of surgical treatment of lung metastasis in soft tissue sarcoma. Arch Surg 142: 70-75, 2007.

9. Rusch VW: Pulmonary metastasectomy. Current indications. Chest 107 (6 Suppl): 322S-331S, 1995.

10. Lin AY, Kotova S, Yanagawa J, Elbuluk O, Wang G, Kar N, Elashoff D, Grogan T, Cameron RB, Singh A, et al: Risk stratification of patients undergoing pulmonary metastasectomy for soft tissue and bone sarcomas. J Thorac Cardiovasc Surg 149: 85-92, 2015.

11. Casson AG, Putnam JB, Natarajan G, Johnston DA, Mountain C, McMurtrey $M$ and Roth JA: Five-year survival after pulmonary metastasectomy for adult soft tissue sarcoma. Cancer 69 662-668, 1992.
12. Meyer WH, Schell MJ, Kumar AP, Rao BN, Green AA, Champion J and Pratt CB: Thoracotomy for pulmonary metastatic osteosarcoma. An analysis of prognostic indicators of survival. Cancer 59: 374-379, 1987.

13. Thomford NR, Woolner LB and Clagett OT: The surgical treatment of metastatic tumors in the lungs. J Thorac Cardiovasc Surg 49: 357-363, 1965.

14. Smith R, Pak Y, Kraybill W and Kane JM III: Factors associated with actual long-term survival following soft tissue sarcoma pulmonary metastasectomy. Eur J Surg Oncol 35: 356-361, 2009.

15. Garcia Franco CE, Torre W, Tamura A, Guillen-Grima F, San-Julian M, Martin-Algarra S and Pardo FJ: Long-Term results after resection for bone sarcoma pulmonary metastases. Eur J Cardiothorac Surg 37: 1205-1208, 2010.

16. Kim S, Ott HC, Wright CD, Wain JC, Morse C, Gaissert HA Donahue DM, Mathisen DJ and Lanuti M: Pulmonary resection of metastatic sarcoma: Prognostic factors associated with improved outcomes. Ann Thorac Surg 92: 1780-1786, 2011.

17. Mizuno T, Taniguchi T, Ishikawa Y, Kawaguchi K, Fukui T, Ishiguro F, Nakamura $\mathrm{S}$ and Yokoi K: Pulmonary metastasectomy for osteogenic and soft tissue sarcoma: Who really benefits from surgical treatment? Eur J Cardiothorac Surg 43: 795-799, 2013.

This work is licensed under a Creative Commons Attribution-NonCommercial-NoDerivatives 4.0 International (CC BY-NC-ND 4.0) License. 